\title{
DIVERSITY COMBINING WITH UP-LINK POWER CONTROL
}

\author{
Sung Sik Nam \\ Department of Electrical and \\ Computer Engineering \\ Texas A\&M University \\ College station, Texas \\ E-mail: ssnam11@tamu.edu
}

\author{
Mohamed-Slim Alouini \\ Department of Electrical Engineering \\ Texas A\&M University at Qatar \\ Education City \\ Doha, Qatar \\ E-mail: alouini@qatar.tamu.edu
}

\author{
Khalid A. Qaraqe \\ Department of Electrical Engineering \\ Texas A\&M University at Qatar \\ Education City \\ Doha, Qatar \\ E-mail: kqaraqe@tamu.edu
}

\section{ABSTRACT}

We introduce in this paper a new adaptive power-controlled diversity combining scheme that reduces the average transmitted power of the mobile units while meeting a certain minimum required quality of service. The key idea is (i) to collect and combine all the available diversity paths at the base station and then (ii) to request the mobile unit to increase or decrease its transmitted power just to track the required target signal-tonoise ratio (SNR). Four power control variants accounting for practical implementation constraints including discrete power levels and transmitter gain saturation are proposed and studied. Some selected numerical results, show that the proposed scheme offers considerable savings in the transmitted power levels over a wide SNR range but amplifier saturation leads to a violation of the target BER requirement in the low average SNR range. Additional numerical examples, show that the power control variants that take into account practical implementation constraints conserve the main features of the ideal continuous power algorithm.

\section{INTRODUCTION}

Wireless communication systems are subject to a harsh propagation environment which leads to frequent fading dips in the received signal. These tough conditions and resulting fading dips make reliable communication very hard. As such various fading countermeasure techniques are needed to improve the performance of these systems. For instance power control and diversity combining are typically used in existing and emerging wireless communication systems to mitigate the problem of signal power fading. In this paper, inspired by the mode of operation of power control algorithms in the 3GPP standard [1], we propose and study an up-link power controlled diversity combining (UPC-DC) scheme. As its name indicates it, UPC-DC combines the features of classical diversity combining with some up-link power control from the mobile unit (MU) to the base station (BS). UPC-DC capitalizes first on diversity combining by collecting and combining all the available diversity paths at the BS. Subsequently and based on the resulting combined signal-to-noise ratio (SNR), the BS requests via a feedback path the MU to increase or decrease its transmitted power just to track a particular required target SNR. In this paper, we study the performance of the proposed scheme and show how this scheme reduces the average bit-error-rate (BER) with, of course, an attendant (and quantifiable) small increase in transmitted power only in the low average SNR range.

The remainder of the paper is organized as follows. Sec- tion II. presents the system and channel models then gives the details behind the mode of operation of various variants of UPC-DC. While section III. provides some analytical results, section IV. illustrates these results via some selected figures. Finally, section V. offers some concluding remarks.

\section{Models AND Mode OF Operation}

\section{A. System Model}

We consider a generic diversity system with $L$ available diversity paths. This includes for example, RAKE receivers which are used in wideband CDMA systems to combine the available resolvable multipaths. For hardware complexity considerations, we assume that up to $L_{c}$ branches can be combined at the receiver side (i.e., the number of fingers of the RAKE receiver is limited to $L_{c}$ ). We also assume, that the proposed UPC-DC scheme has a reliable feedback path between the receiver and the transmitter and is implemented in a discretetime fashion. More specifically, and as shown in Fig. 1, short guard periods are periodically inserted into the transmitted signal. During these guard periods, the receiver performs a series of operation, including (i) path estimation, (ii) combined SNR comparison with respect to the predetermined SNR threshold, and (iii) request to the MU power amplifier to increase or decrease its gain by a specific amount. Once all the available diversity paths are selected and once the appropriate transmitted power is reached, the combiner (at the receiver end) and the power amplifier (at the transmitter) are configured accordingly and this transmitter and receiver settings are used throughout the subsequent data burst.

\section{B. Channel Model}

We denote by $\gamma_{l}(l=1,2, \cdots, L)$, the received SNR of the $l$ th diversity path (under nominal transmitted power from the MU ${ }^{1}$ ) and, as illustrated in Fig. 1, we adopt a block flat fading channel model. More specifically, assuming slowlyvarying fading conditions, the different diversity paths experience roughly the same fading conditions (or equivalently the same SNR) during the data burst and its preceding guard period. In addition, the fading conditions are assumed to (i) be independent across the diversity paths and between different guard period and data burst pairs, and (ii) follow anyone of the popular fading models such as Rayleigh, Rice, or Nakagami$m$.

${ }^{1}$ The MU nominal transmitted power is assumed to correspond to an initial level of output power that is adjusted/set to minimize the average outer cell interference in a particular deployment. 


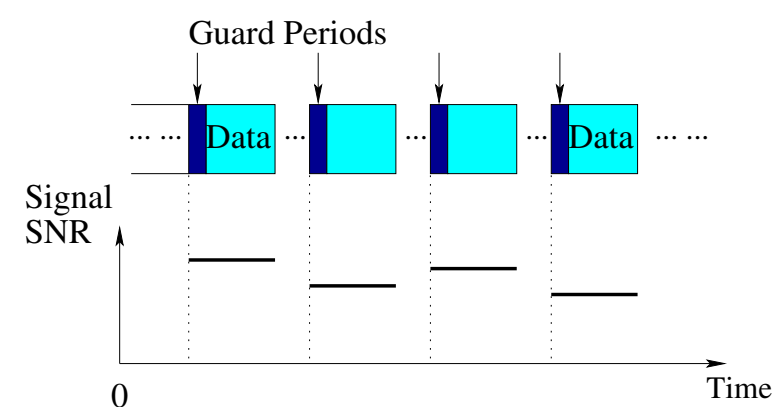

Figure 1: Block fading channel model.

\section{Mode of Operation of UPC-DC}

If the number $L$ of available paths in the BS is below the number $L_{c}$ of paths that can be combined, the BS combines all the available paths as per the rules of maximal-ratio combining (MRC). On the other hand, if the number $L$ of available diversity paths exceeds the number $L_{c}$ of paths that can be combined, the BS uses generalized selection combining (GSC) (see for example [2-5]). With GSC, the $L_{c}$ (among the $L$ available) diversity branches with the best quality (quantified for example in terms of fading amplitude or equivalently instantaneous SNR)) are selected and combined in an MRC fashion. At the beginning of the guard period, the MU power amplifier gain $G$ (with respect to the nominal transmitted power) is initially set to $0 \mathrm{~dB}$ and based on this setting the combining process described above is performed. If the combiner fails to meet the $\gamma_{T}$ requirement during the combining process phase, the receiver activates the power control mechanism and requests the transmitter to increase its gain in order to meet the target SNR requirement. If on the other hand, the required output SNR $\gamma_{T}$ is reached during this initial phase, then the receiver activates also the power control mechanism but requests in this case the transmitter to decrease its gain such that the output combined SNR just matches the target SNR requirement. We consider in our study, four power adaptation variants:

1. Continuous Adaptation without Amplifier Gain Saturation In this first ideal case, we assume that the gain of the MU amplifier $G$ can be adjusted in a continuous fashion and is not limited by any maximal value.

2. Continuous Adaptation with Amplifier Gain Saturation In this case, we still assume that the gain of the transmitter amplifier can be adjusted continuously but saturates to a certain maximal value $G_{\max }$. In the case, that a gain beyond $G_{\max }$ is needed to meet the required target SNR, we assume that the MU units des-activates the power control mechanism and transmits with the nominal power level. This is done to save some valuable battery lifetime but comes of course at the expense of the violation of the target SNR requirement in very adverse channel fading conditions.

3. Discrete Adaptation without Amplifier Gain Saturation Similar to the power control algorithms that are implemented in the 3 GPP standard, we assume in this case that the MU amplifier gain can only take discrete values. This gain can be adjusted using a binary feedback and a power control step size $G_{\delta}$.

4. Discrete Adaptation with Amplifier Gain Saturation In this most practical case, we assume that the gain takes discrete values and saturates to a fixed maximal value $G_{\max }$. We again assume that the MU transmits with nominal power level if a gain beyond $G_{\max }$ is needed to meet the required target $\mathrm{SNR}$.

\section{PERFORMANCE ANALYSis}

\section{A. Statistics of the Combined SNR}

Regardless of the type of adaptation used, the probability density function (PDF), $p_{\gamma_{c}}(\cdot)$, of the combined SNR at the end of the the diversity combining stage is given by

$$
p_{\gamma_{c}}(\gamma)=\left(1-P_{L}\left(L_{c}\right)\right) p_{\gamma_{\mathrm{mrc}}}(\gamma)+P_{L}\left(L_{c}\right) p_{\gamma_{\mathrm{gsc}}}(\gamma),
$$

where $P_{L}(l)=P[L \leq l]$ is the cumulative distribution function (CDF) of the number of available diversity paths in the area of deployment which can be for example modeled by a Poisson distribution $[6,7], p_{\gamma_{\mathrm{mrc}}}(\cdot)$ is the PDF at the output of an $L$-branch MRC diversity combiner which is known in closedform for many fading scenarios of interest [8], and $p_{\gamma_{\mathrm{gsc}}}(\cdot)$ is the PDF at the output of a GSC receiver combining the $L_{c}$ strongest branches among the $L$ available ones which is also known in closed-form for many fading scenarios of interest [8].

With the PDF of the combined SNR at hand, we can find the average BER and the additional average $\mathrm{dB}$ gain $G^{2}$ that is required by the uplink power control for the four power adaptation variants under consideration. Due to the space limitation, we omit in what follows the detailed derivations for these quantities and we just provide the final analytical formulas as well as some selected numerical results illustrating the performance of our proposed UPC-DC.

\section{B. Continuous Adaptation without Amplifier Gain Saturation}

In this case, the average BER is constant and equal to $\operatorname{BER}\left(\gamma_{T}\right)$, where $\operatorname{BER}(\gamma)$ is the BER of the modulation when used over an additive white Gaussian noise (AWGN) channel with SNR $\gamma$. The corresponding additional average $\mathrm{dB}$ gain can be shown to be given by

$$
G_{\mathrm{dB}}=\gamma_{T_{\mathrm{dB}}}-10 \int_{0}^{\infty} \log _{10}(\gamma) p_{\gamma_{c}}(\gamma) d \gamma .
$$

For independent identically distributed (i.i.d.) Rayleigh fading conditions and $L_{c} \geq L$ (i.e. the receiver combines the $L$ available diversity paths in an MRC fashion and $p_{\gamma_{c}}(\gamma)=$ $\left.p_{\gamma_{\mathrm{mrc}}}(\gamma)\right)$, it can be shown with the help of [9, Eq. (4.352.1)] and $[9$, Eq. (8.365.4)] that

$$
G_{\mathrm{dB}}=\gamma_{T_{\mathrm{dB}}}-\frac{10}{\ln 10}\left(-C+\sum_{l=1}^{L-1} \frac{1}{l}+\ln \bar{\gamma}\right),
$$

where $C=0.577215664$ is the Euler constant.

\footnotetext{
${ }^{2}$ The additional average $\mathrm{dB}$ gain $G_{\mathrm{dB}}$ is defined as the average of the additional $\mathrm{dB}$ gain and is therefore given by $G_{\mathrm{dB}}=E\left[\gamma_{T_{\mathrm{dB}}}-\gamma_{c_{\mathrm{dB}}}\right]$
} 
C. Continuous Adaptation with Amplifier Gain Saturation In this case, the average BER can be shown to be given by

$$
\begin{aligned}
\mathrm{BER} & =\int_{0}^{\gamma_{T} / G_{\max }} \operatorname{BER}(\gamma) p_{\gamma_{c}}(\gamma) d \gamma+ \\
& +\operatorname{BER}\left(\gamma_{T}\right)\left(1-P_{\gamma_{c}}\left(\gamma_{T} / G_{\max }\right)\right)
\end{aligned}
$$

where $P_{\gamma_{c}}(\cdot)$ is the CDF of the combined SNR at the end of the combining phase. For i.i.d. Rayleigh fading conditions and $L_{c} \geq L$, it can be shown that

$$
\begin{aligned}
\mathrm{BER} & =0.5\left[-\operatorname{erfc}(\sqrt{\gamma}) \frac{\Gamma\left(L, \frac{\gamma}{\gamma}\right)}{(L-1) !}\right]_{0}^{\frac{\gamma_{T}}{G_{\max }}} \\
& +0.5 \sum_{l=0}^{L-1} \frac{1}{\sqrt{\pi} l !}\left(\frac{1}{\bar{\gamma}}\right)^{l} \mu^{-\left(l+\frac{1}{2}\right)}\left[\Gamma\left(l+\frac{1}{2}, \mu \gamma\right)\right]_{0}^{\frac{\gamma_{T}}{G_{\max }}} \\
& +0.5 \operatorname{erfc}\left(\sqrt{\gamma_{T}}\right) \frac{\Gamma\left(L, \frac{\gamma_{T}}{\gamma G_{\max }}\right)}{(L-1) !}
\end{aligned}
$$

where $\mu=1+\frac{1}{\bar{\gamma}}, \Gamma(\cdot, \cdot)$ is the incomplete gamma function defined for positive integer $n$ by [9, Eq. (8.352.2)]

$$
\Gamma(n, x)=(n-1) ! e^{-x} \sum_{m=0}^{n-1} \frac{x^{m}}{m !}
$$

and for general real $\alpha$ as [9, Eq. (8.350.2)]

$$
\Gamma(\alpha, x)=\int_{x}^{+\infty} t^{\alpha-1} e^{-t} d t
$$

and $\operatorname{erfc}(\cdot)$ is the complementary error function defined in [9, Eq. (8.350.2)] as

$$
\operatorname{erfc}(x)=\frac{2}{\sqrt{\pi}} \int_{x}^{+\infty} e^{-t^{2}} d t
$$

The corresponding additional average $\mathrm{dB}$ gain can be shown to be given by

$$
\begin{aligned}
G_{\mathrm{dB}} & =\gamma_{T_{\mathrm{dB}}}\left(1-P_{\gamma_{c}}\left(\gamma_{T} / G_{\max }\right)\right) \\
& -10 \int_{\frac{\gamma_{T}}{G_{\max }}}^{+\infty} \log _{10}(\gamma) p_{\gamma_{c}}(\gamma) d \gamma
\end{aligned}
$$

For i.i.d. Rayleigh fading conditions and $L_{c} \geq L$, it can be shown that

$$
\begin{aligned}
G_{\mathrm{dB}} & =\frac{\gamma_{T_{\mathrm{dB}}}}{(L-1) !} \Gamma\left(L, \frac{\gamma_{T}}{\bar{\gamma} G_{\max }}\right) \\
& -\frac{10}{\ln 10(L-1) !}\left(\frac{\gamma_{T}}{\bar{\gamma} G_{\max }}\right)^{L} \ln \left(\frac{\gamma_{T}}{G_{\max }}\right) \Gamma\left(L, \frac{\gamma_{T}}{\bar{\gamma} G_{\max }}\right) \\
& -\frac{10}{\ln 10} \sum_{l=0}^{L-1} \frac{\Gamma\left(l, \frac{\gamma_{T}}{\bar{\gamma} G_{\max }}\right)}{l !} .
\end{aligned}
$$

\section{Discrete Adaptation without Amplifier Gain Saturation} In this case, the average BER can be shown to be given by

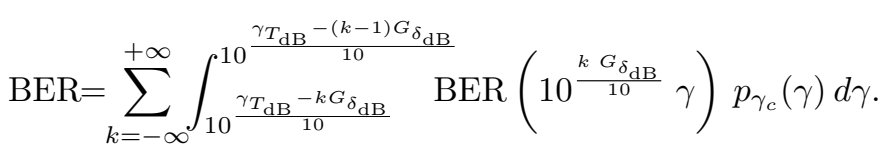

For i.i.d. Rayleigh fading conditions and $L_{c} \geq L$, it can be shown that

$$
\begin{aligned}
\mathrm{BER} & =\sum_{k=-\infty}^{+\infty} 0.5\left[-\operatorname{erfc}(\sqrt{a(k) \gamma}) \frac{\Gamma\left(L, \frac{\gamma}{\gamma}\right)}{(L-1) !}\right. \\
& \left.+\sum_{l=0}^{L-1} \sqrt{\frac{a(k) 1}{\pi l !}}\left(\frac{1}{\bar{\gamma}}\right)^{l} \beta^{-\left(l+\frac{1}{2}\right)} \Gamma\left(l+\frac{1}{2}, \beta \gamma\right)\right]_{10}^{\frac{\gamma_{T_{\mathrm{dB}}-k G_{\delta}}}{10}},
\end{aligned}
$$

where $a(k)=10^{\frac{k G_{\delta}}{10}}$ and $\beta=a(k)+\frac{1}{\bar{\gamma}}$.

The corresponding additional average $\mathrm{dB}$ gain can be shown to be given by

$$
G_{\mathrm{dB}}=\sum_{k=-\infty}^{+\infty} P_{\gamma_{c}}\left(10^{\frac{\gamma_{T_{\mathrm{dB}}}-(k-1) G_{\delta_{\mathrm{dB}}}}{10}}\right) G_{\delta_{\mathrm{dB}}}
$$

For i.i.d. Rayleigh fading conditions and $L_{c} \geq L$, it can be shown that

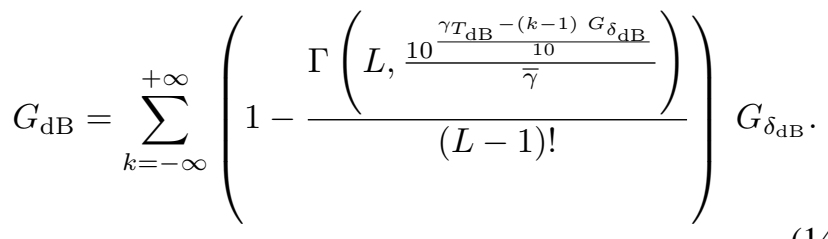

\section{E. Discrete Adaptation with Amplifier Gain Saturation} In this case, the average BER can be shown to be given by

$$
\begin{aligned}
\mathrm{BER} & =\sum_{k=-\infty}^{+K_{M}} \int_{10}^{10 \frac{\gamma_{T_{\mathrm{dB}}}-(k-1) G_{\delta_{\mathrm{dB}}}}{10}} \operatorname{BER}\left(10 \frac{\gamma_{T_{\mathrm{dB}}}-k G_{\delta_{\mathrm{dB}}}}{10}\right. \\
& +\int_{0}^{\gamma_{T} / G_{\max }} \operatorname{BER}(\gamma) p_{\gamma_{c}}(\gamma) d \gamma
\end{aligned}
$$

where $K_{M}=G_{\max _{\mathrm{dB}}} / G_{\delta_{\mathrm{dB}}}$. For i.i.d. Rayleigh fading conditions and $L_{c} \geq L$, it can be shown that

$\mathrm{BER}=\sum_{k=-\infty}^{K_{M}} 0.5\left[-\operatorname{erfc}(\sqrt{a(k) \gamma}) \frac{\Gamma\left(L, \frac{\gamma}{\bar{\gamma}}\right)}{(L-1) !}\right.$

$\left.+\sum_{l=0}^{L-1} \sqrt{\frac{a(k) 1}{\pi l !}}\left(\frac{1}{\bar{\gamma}}\right)^{l} \beta^{-\left(l+\frac{1}{2}\right)} \Gamma\left(l+\frac{1}{2}, \beta \gamma\right)\right]_{10}^{10^{\frac{\gamma_{T_{\mathrm{dB}}}-(k-1) G_{\delta}}{10}}}$

$+0.5\left[-\operatorname{erfc}(\sqrt{\gamma}) \frac{\Gamma\left(L, \frac{\gamma}{\bar{\gamma}}\right)}{(L-1) !}\right]_{0}^{\frac{\gamma_{T}}{G_{\max }}}$ 


$$
+0.5 \sum_{l=0}^{L-1} \frac{1}{\sqrt{\pi} l !}\left(\frac{1}{\bar{\gamma}}\right)^{l} \mu^{-\left(l+\frac{1}{2}\right)}\left[\Gamma\left(l+\frac{1}{2}, \mu \gamma\right)\right]_{0}^{\frac{\gamma_{T}}{G_{\max }}}
$$

The corresponding additional average $\mathrm{dB}$ gain can be shown to be given by

$$
G_{\mathrm{dB}}=\sum_{k=-\infty}^{K_{M}+1} f(k) P_{\gamma_{c}}\left(10^{\frac{\gamma_{T_{\mathrm{dB}}}-(k-1) G_{\delta_{\mathrm{dB}}}}{10}}\right) G_{\delta_{\mathrm{dB}}}
$$

where

$$
f(k)= \begin{cases}1 & k=-\infty, \cdots, K_{M} \\ -K_{M} & k=K_{M}+1 .\end{cases}
$$

For i.i.d. Rayleigh fading conditions and $L_{c} \geq L$, it can be shown that

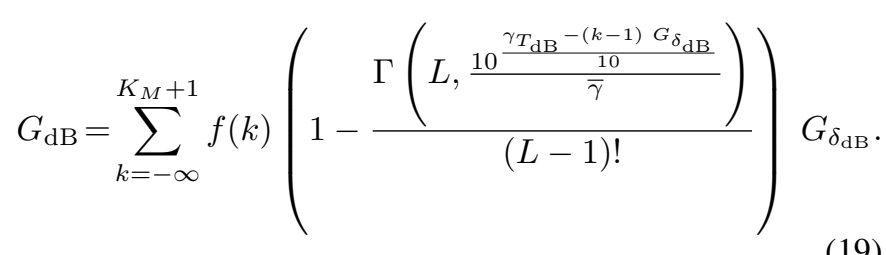

\section{NUMERICAL EXAMPLES}

Fig. 2 compares the BER of binary phase shift keying (BPSK) when used with MRC and no power control and when used in conjunction with continuous UPC-MRC with and without amplifier gain saturation. Clearly UPC makes the system just meet the target BER over the whole SNR range while systems without PC either fails to meet this target BER in the low average SNR region or exceeds it in the high average SNR region. In addition, when UPC is used the saturation of the transmitter amplifier leads to a violation of the target BER requirement in the low average SNR region. However, we can see from Fig. 3 that a peak power constraint at the transmitter side leads to a considerable decrease in the required additional average transmitter gain in this same low average SNR region.

While Figs. 4 and 5 compare continuous and discrete power adaptation (with different step sizes) without amplifier gain saturation, Figs. 6 and 7 do the same comparison when there exists a peak power constraint at the transmitter side. One can see from these figures, that as long as $G_{\max _{\mathrm{dB}}}$ is an integer multiple of $G_{\delta_{\mathrm{dB}}}$ discrete power control requires a slightly higher average gain but offers correspondingly a decrease in the average BER over the whole average SNR range. However if the the value of $G_{\max _{\mathrm{dB}}}$ is not an integer multiple of $G_{\delta_{\mathrm{dB}}}$, this behavior is reversed in the low average SNR range.

\section{CONCLUSION}

We introduced in this paper a new adaptive up-link diversity combining scheme. The key idea is to take advantage of all the diversity offered by the channel and then request the transmitter (i) to increase its power level during very adverse channel conditions in order to reach the target SNR and (ii) to decrease its power level during favorable channel conditions just to keep the SNR level at the target required SNR. Four power control variants accounting for practical implementation constraints in(16)cluding discrete power levels and transmitter gain saturation were proposed and studied. Some selected numerical results, show that the scheme offers considerable savings in the transmitted power levels over a wide SNR range but amplifier saturation leads to a violation of the target BER requirement in the low average SNR range. Additional numerical examples, show that the power control variants that take into account practical implementation constraints conserve the main features of the ideal continuous power algorithm.

\section{ACKNOWLEDGMENT}

This work was supported in part by the Qatar Foundation for Education, Sciences, and Community Development, Doha, Qatar and in part by Qatar Telecom (Qtel), Qatar.

\section{REFERENCES}

[1] "3 GPP technical specification group radio access network: Physical layer procedures (FDD) specifications, 1999.” TS 25.214 V4.6.0 (2003-03).

[2] N. Kong, T. Eng, and L. B. Milstein, "A selection combining scheme for RAKE receivers," in Proc. IEEE Int. Conf. Univ. Personal Comm. ICUPC'95, Tokyo, Japan, pp. 426-429, November 1995.

[3] M. Z. Win and Z. A. Kostić, "Virtual path analysis of selective Rake receiver in dense multipath channels," IEEE Commun. Letters, vol. 3, pp. 308-310, November 1999.

[4] M. -S. Alouini and M. K. Simon, "An MGF-based performance analysis of generalized selective combining over Rayleigh fading channels," IEEE Trans. Commun., vol. COM-48, pp. 401-415, March 2000.

[5] Y. Roy, J. -Y. Chouinard, and S. Mahmoud, "Selection diversity combining with multiple antennae for mm-wave indoor wireless channels," IEEE Journal of Selected Areas in Communications, vol. SAC-14, pp. 674-682, May 1996.

[6] G. L. Turin, F. D. Clapp, T. L. Johnston, S. B. Fine, and D. Lavry, "A statistical model of urban multipath propagation," IEEE Trans. Veh. Technol., vol. VT-21, pp. 1-9, February 1972.

[7] L. Yuanqing, "A theoretical formulation for the distribution density of multipath delay spread in a land mobile radio environment," IEEE Trans. Veh. Technol., vol. VT-43, pp. 379-388, May 1994.

[8] M. K. Simon and M. -S. Alouini, "Digital communications over generalized fading channels.” New York, NY: John Wiley \& Sons, 2004.

[9] I. S. Gradshteyn and I. M. Ryzhik, Table of Integrals, Series, and Products. San Diego, CA: Academic Press, fifth ed., 1994. 


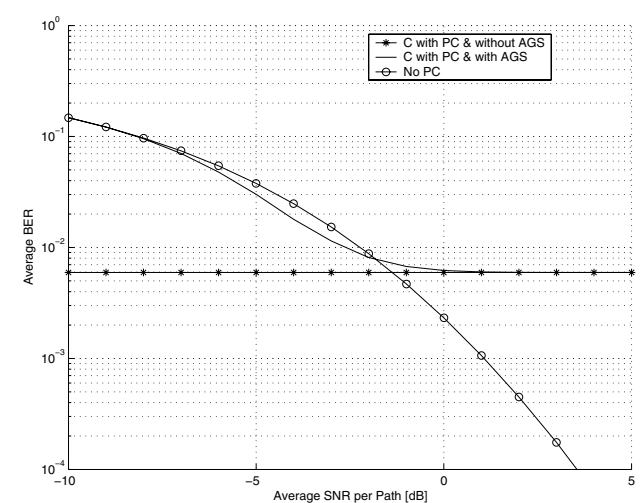

Figure 2: Average BER of B-PSK with (i) continuous UPCMRC and no amplifier gain saturation, (ii) continuous UPCMRC and amplifier gain saturation, and (iii) $\mathrm{MRC}$ and no power control $\left(L_{c}=L=6, \gamma_{T}=5 \mathrm{~dB}\right.$, and $G_{\max }=3$ $\mathrm{dB})$.

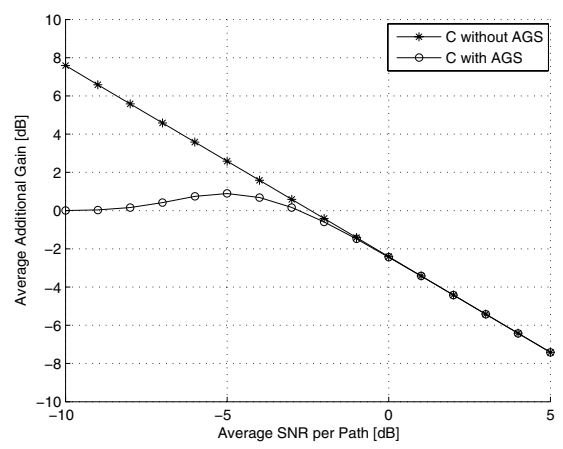

Figure 3: Average additional $\mathrm{dB}$ gain of the transmitter amplifier using continuous UPC-DC with and without saturation $\left(L_{c}=L=6, \gamma_{T}=5 \mathrm{~dB}\right.$, and $\left.G_{\max }=3 \mathrm{~dB}\right)$.

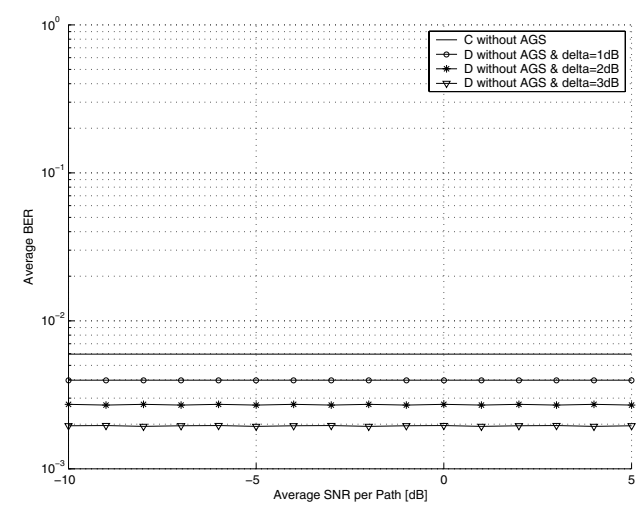

Figure 4: Average BER of B-PSK with (i) continuous UPCMRC and no amplifier gain saturation and (ii) discrete UPCMRC and no amplifier gain saturation $\left(L_{c}=L=6\right.$ and $\gamma_{T}=$ $5 \mathrm{~dB})$.

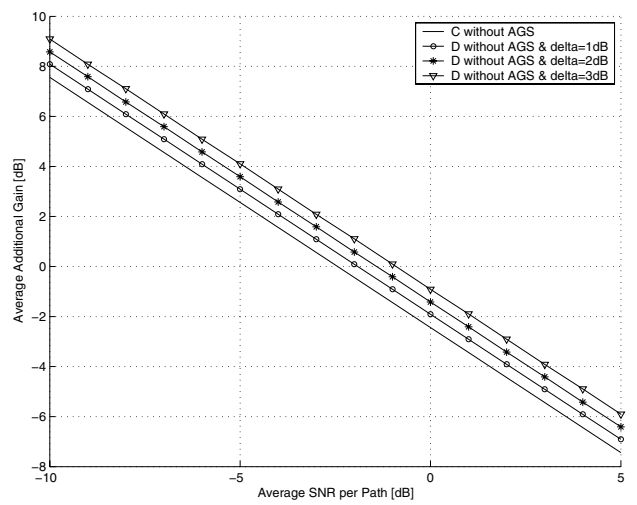

Figure 5: Average additional $\mathrm{dB}$ gain of the transmitter amplifier with (i) continuous UPC-MRC and no amplifier gain saturation and (ii) discrete UPC-MRC and no amplifier gain saturation $\left(L_{c}=L=6\right.$ and $\left.\gamma_{T}=5 \mathrm{~dB}\right)$.

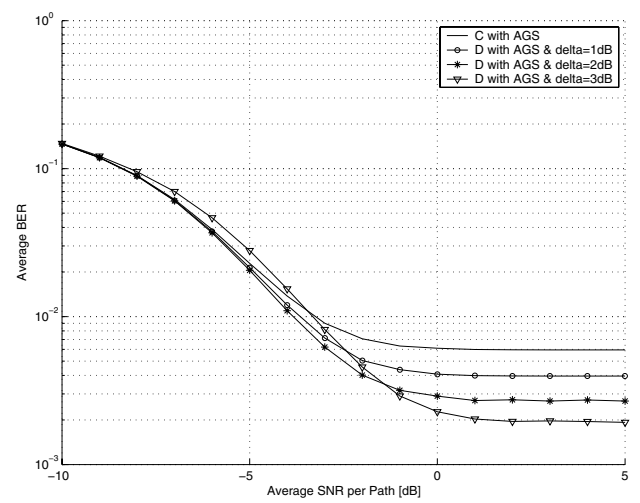

Figure 6: Average BER of B-PSK with (i) continuous UPCMRC and amplifier gain saturation and (ii) discrete UPC-MRC and amplifier gain saturation $\left(L_{c}=L=6, \gamma_{T}=5 \mathrm{~dB}\right.$, and $\left.G_{\max }=4 \mathrm{~dB}\right)$.

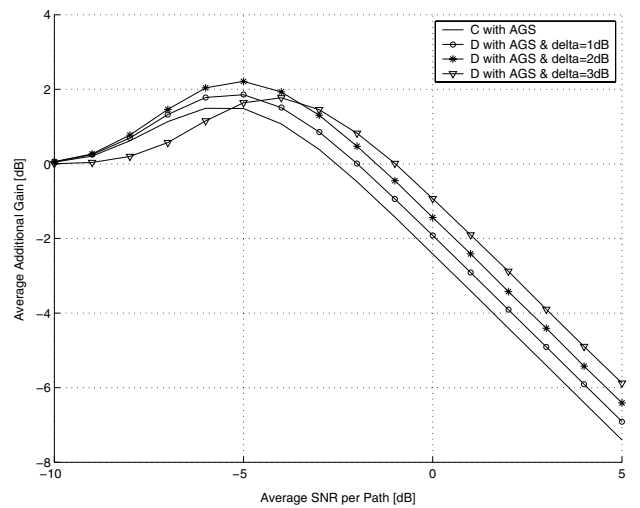

Figure 7: Average additional dB gain of the transmitter amplifier with (i) continuous UPC-MRC and amplifier gain saturation and (ii) discrete UPC-MRC and amplifier gain saturation $\left(L_{c}=L=6, \gamma_{T}=5 \mathrm{~dB}\right.$, and $\left.G_{\max }=4 \mathrm{~dB}\right)$. 